\title{
Erratum to: Multipliers and Morrey Spaces
}

\author{
Pierre Gilles Lemarié-Rieusset
}

Published online: 19 June 2014

(C) Springer Science+Business Media Dordrecht 2014

\section{Erratum to: Potential Anal (2013) 38:741-752 \\ DOI 10.1007/s11118-012-9295-8}

We correct the complex interpolation results for Morrey spaces which is false for the first interpolation functor of Calderón, but is exact for Calderón's second interpolation functor. Furthermore, the author's affiliation has changed. The updated affiliation is provided below.

In my paper "Multipliers and Morrey spaces" [3], there is a slight mistake in Theorem 3, concerning the interpolation of Morrey spaces.

Let $\mathcal{B}$ be the collection of all Euclidean balls $B$ on $\mathbb{R}^{d}: B=B\left(x_{B}, r_{B}\right)=\left\{x \in \mathbb{R}^{d} / \mid x-\right.$ $x_{B} \mid<R_{B}$. For $B \in \mathcal{B}$, we write $|B|=\int_{B} d x=|B(0,1)| r_{B}^{d}$. Define, for $1<p \leq q<$ $+\infty$, the space $\dot{M}^{p, q}\left(\mathbb{R}^{n}\right)$ as the space of locally $p$-integrable functions $f$ such that

$$
\|f\|_{\dot{M}^{p, q}}:=\sup _{B \in \mathcal{B}}|B|^{1 / q-1 / p}\left(\int_{B}|f(x)|^{p} d x\right)^{1 / p}<+\infty .
$$

It is easy to check that, when $1<p_{0} \leq q_{0}<+\infty$ and $1<p_{1} \leq q_{1}<+\infty$, and

$$
\left(\frac{1}{p}, \frac{1}{q}\right)=(1-\theta)\left(\frac{1}{p_{0}}, \frac{1}{q_{0}}\right)+\theta\left(\frac{1}{p_{1}}, \frac{1}{q_{1}}\right)
$$

for some $\theta \in(0,1)$, then $\dot{M}^{p_{0}, q_{0}} \cap \dot{M}^{p_{1}, q_{1}} \subset \dot{M}^{p, q}$ and

$$
\|f\|_{\dot{M}^{p, q}} \leq\|f\|_{\dot{M}^{p_{0}, q_{0}}}^{1-\theta}\|f\|_{\dot{M}^{p_{1}, q_{1}}}^{\theta}
$$

The question I studied was then whether one may find an interpolation functor $F$ of exponent $\theta$ such that $F\left(\dot{M}^{p_{0}, q_{0}}, \dot{M}^{p_{1}, q_{1}}\right)=\dot{M}^{p, q}$. If so, one should have the continuous embeddings

$$
\left[\dot{M}^{p_{0}, q_{0}}, \dot{M}^{p_{1}, q_{1}}\right]_{\theta, 1} \subset \dot{M}^{p, q} \subset\left[\dot{M}^{p_{0}, q_{0}}, \dot{M}^{p_{1}, q_{1}}\right]_{\theta, \infty}
$$

The online version of the original article can be found at http://dx.doi.org/10.1007/s11118-012-9295-8.

P. G. Lemarié-Rieusset $(\bowtie)$

Laboratoire de Mathématiques et Modélisation d' Évry, UMR CNRS 8071, Université d' Évry, Évry, France

e-mail: plemarie@univ-evry.fr 
The inclusion $\left[A_{0}, A_{1}\right]_{\theta, 1} \subset F\left(A_{0}, A_{1}\right)$ is a direct conclusion from the inequality

$$
\|f\|_{F\left(A_{0}, A_{1}\right)} \leq C\|f\|_{A_{0}}^{1-\theta}\|f\|_{A_{1}}^{\theta}
$$

(obtained by interpolation inequalities for the operator norms of $\lambda \mapsto \lambda f$ from $\mathbb{R}$ to $A_{0}$ and from $\mathbb{R}$ to $A_{1}$, hence from $\mathbb{R}$ to $\left.F\left(A_{0}, A_{1}\right)\right)$. The inclusion $F\left(A_{0}, A_{1}\right) \subset\left[A_{0}, A_{1}\right]_{\theta, \infty}$ is proven in [1] under the assumption that $A_{0} \cap A_{1}$ is dense in $A_{0}$ and in $A_{1}$. This is not the case for Morrey spaces. However, one may easily adapt the proof, as Morrey spaces are dual spaces (see for instance [6]).

If we assume that $A_{0}=B_{0}^{\prime}$ and $A_{1}=B_{1}^{\prime}$ and that $B_{0} \cap B_{1}$ is dense in $B_{0}$ and $B_{1}$; then, for $b \in B_{0} \cap B_{1}$, the linear form $T_{b}: f \mapsto\langle f \mid b\rangle$ has a norm less than $\|b\|_{B_{0}}$ as an operator from $A_{0}$ to $\mathbb{R}$ and less than $\|b\|_{B_{1}}$ as an operator from $A_{1}$ to $\mathbb{R}$, hence as a norm less than $C\|b\|_{B_{0}}^{1-\theta}\|b\|_{B_{1}}^{\theta}$; thus, $\tilde{T}_{f}: b \mapsto\langle f \mid b\rangle$ is a continuous linear form on $\left[B_{0}, B_{1}\right]_{\theta, 1}$. This gives that $f \in\left(\left[B_{0}, B_{1}\right]_{\theta, 1}\right)^{\prime}=\left[B_{0}^{\prime}, B_{1}^{\prime}\right]_{\theta, \infty}$ (since $B_{0} \cap B_{1}$ is dense in $B_{0}$ and $B_{1}$ ).

The theorem I proved in [3] is the following one:

Theorem 1 Let $1<p_{0} \leq q_{0}<+\infty$ and $1<p_{1} \leq q_{1}<+\infty$, and

$$
\left(\frac{1}{p}, \frac{1}{q}\right)=(1-\theta)\left(\frac{1}{p_{0}}, \frac{1}{q_{0}}\right)+\theta\left(\frac{1}{p_{1}}, \frac{1}{q_{1}}\right)
$$

for some $\theta \in(0,1)$. Then there exists an interpolation functor $F$ of exponent $\theta$ such that $F\left(\dot{M}^{p_{0}, q_{0}}, \dot{M}^{p_{1}, q_{1}}\right)=\dot{M}^{p, q}$ if and only if $p_{0} / q_{0}=p_{1} / q_{1}$.

The negative result for the case $p_{0} / q_{0} \neq p_{1} / q_{1}$ was proven by a generalization of a counterexample by Ruiz and Vega [4] which proves that, in that case, we don't have the embedding of $\dot{M}^{p, q}$ into $\left[\dot{M}^{p_{0}, q_{0}}, \dot{M}^{p_{1}, q_{1}}\right]_{\theta, \infty}$.

The proof for the positive result (on the case $p_{0} / q_{0}=p_{1} / q_{1}$ ) was inexact. I claimed that in that case we have the complex interpolation $\dot{M}^{p, q}=\left[\dot{M}^{p_{0}, q_{0}}, \dot{M}^{p_{1}, q_{1}}\right]_{\theta}$. But this is false as pointed to me by Sickel (who has recently characterized the intermediate space $\dot{M}^{p, q} \subset$ $\left[\dot{M}^{p_{0}, q_{0}}, \dot{M}^{p_{1}, q_{1}}\right]_{\theta}$ in a joint work with Yang and Yuan [5]). Indeed, it is easy to see that, when $p_{0} / q_{0}=p_{1} / q_{1}=p_{0} / q_{0}<1$ and $p_{0} \neq p_{1}, \dot{M}^{p_{0}, q_{0}} \cap \dot{M}^{p_{1}, q_{1}}$ is not dense in $\dot{M}^{p, q}$, while it is always true that $A_{0} \cap A_{1}$ is dense in $\left[A_{0}, A_{1}\right]_{\theta}$ (see [1]). Sickel's counterexample is very clear : if $r=\min \left(p_{0}, p_{1}\right)$ and $s=\max \left(q_{0}, q_{1}\right)$, we have $\dot{M}^{p, q} \subset \dot{M}^{r, q}$ and $\dot{M}^{p_{0}, q_{0}} \cap$ $\dot{M}^{p_{1}, q_{1}} \subset \dot{M}^{r, s}$; thus the applications $f \mapsto \rho^{d(1 / q-1 / r)} 1_{B(0, \rho)} f$ are equicontinuous from $\dot{M}^{p, q}$ to $L^{r}$; for $f \in \dot{M}^{p_{0}, q_{0}} \cap \dot{M}^{p_{1}, q_{1}}$, we have $\lim _{\rho \rightarrow 0} \rho^{d(1 / q-1 / r)}\left\|1_{B(0, \rho)} f\right\|_{r}=0$, while for $f_{0}=|x|^{-d / q} \in \dot{M}^{p, q}$, we have $\lim _{\rho \rightarrow 0} \rho^{d(1 / q-1 / r)}\left\|1_{B(0, \rho)} f_{0}\right\|_{r}>0$; thus $f_{0}$ does not belong to the closure of $\dot{M}^{p_{0}, q_{0}} \cap \dot{M}^{p_{1}, q_{1}}$

However, a slight modification of the proof of [3] gives the following theorem :

Theorem 2 Let $1<p_{0} \leq q_{0}<+\infty$ and $1<p_{1} \leq q_{1}<+\infty$, and

$$
\left(\frac{1}{p}, \frac{1}{q}\right)=(1-\theta)\left(\frac{1}{p_{0}}, \frac{1}{q_{0}}\right)+\theta\left(\frac{1}{p_{1}}, \frac{1}{q_{1}}\right)
$$

If $p_{0} / q_{0}=p_{1} / q_{1}$, then

$$
\dot{M}^{p, q}=\left[\dot{M}^{p_{0}, q_{0}}, \dot{M}^{p_{1}, q_{1}}\right]^{\theta} .
$$

Let us recall that Calderón [2] defined two complex interpolation functors : $\left[A_{0}, A_{1}\right]_{\theta}$ and $\left[A_{0}, A_{1}\right]^{\theta}$. We have $\left[A_{0}, A_{1}\right]_{\theta} \subset\left[A_{0}, A_{1}\right]^{\theta}$ (with equality when at least one of the two spaces $A_{0}$ and $A_{1}$ is reflexive). 
Proof Let us recall the definition of $\left[A_{0}, A_{1}\right]_{\theta}$ and $\left[A_{0}, A_{1}\right]^{\theta}$.

Let $\Omega$ be the open complex strip $\Omega=\{z \in \mathbb{C} / 0<\Re z<1\}$. $\mathcal{F}$ is the space of functions $F$ defined on the closed complex strip $\bar{\Omega}$ such that:

1. $F$ is continuous and bounded from $\bar{\Omega}$ to $A_{0}+A_{1}$

2. $F$ is analytic from $\Omega$ to $A_{0}+A_{1}$

3. $t \mapsto F(i t)$ is continuous from $\mathbb{R}$ to $A_{0}$, and $\lim _{|t| \rightarrow+\infty}\|F(i t)\|_{A_{0}}=0$

4. $t \mapsto F(1+i t)$ is continuous from $\mathbb{R}$ to $A_{1}$, and $\lim _{|t| \rightarrow+\infty}\|F(1+i t)\|_{A_{0}}=0$

Then

and

$$
f \in\left[A_{0}, A_{1}\right]_{\theta} \Leftrightarrow \exists F \in \mathcal{F}, f=F(\theta)
$$

$$
\|f\|_{\left[A_{0}, A_{1}\right]_{\theta}}=\inf _{f=F(\theta)} \max \left(\sup _{t \in \mathbb{R}}\|F(i t)\|_{A_{0}}, \sup _{t \in \mathbb{R}}\|F(1+i t)\|_{A_{1}}\right) .
$$

On the other hand, $\mathcal{G}$ is the space of functions $G$ defined on the closed complex strip $\bar{\Omega}$ such that :

1. $\frac{1}{1+|z|} G$ is continuous and bounded from $\bar{\Omega}$ to $A_{0}+A_{1}$

2. $G$ is analytic from $\Omega$ to $A_{0}+A_{1}$

3. $t \mapsto G(i t)-G(0)$ is Lipschitz from $\mathbb{R}$ to $A_{0}$

4. $t \mapsto G(1+i t)-G(1)$ is Lipschitz from $\mathbb{R}$ to $A_{1}$

Then

and

$\|f\|_{\left[A_{0}, A_{1}\right]^{\theta}}=\inf _{f=G^{\prime}(\theta)} \max \left(\sup _{t_{1}, t_{2} \in \mathbb{R}}\left\|\frac{G\left(i t_{2}\right)-G\left(i t_{1}\right)}{t_{2}-t_{1}}\right\|_{A_{0}, t_{1}, t_{2} \in \mathbb{R}}\left\|\frac{G\left(1+i t_{2}\right)-G\left(1+i t_{1}\right)}{t_{2}-t_{1}}\right\|_{A_{1}}\right)$.

Let us remark that, for continuous functions, (strong) analyticity is equivalent to weak analyticiy or even *-weak analyticity when $A_{0}$ and $A_{1}$ are dual spaces of $B_{0}$ and $B_{1}$ with $B_{0} \cap B_{1}$ dense in $B_{0}$ and $B_{1}$. Indeed, analyticity is equivalent to the fact that, whenever the closed ball $\bar{B}\left(z_{0}, r\right)$ is contained in $\Omega$ and $\left|w-z_{0}\right|<r$, then $F(w)=$ $\frac{1}{2 i \pi} \int_{\left|z-z_{0}\right|=r} F(z) \frac{d z}{z-w}$. As $F$ is continuous, we have, for $b \in B_{0} \cap B_{1}$,

$$
\left\langle b \mid \frac{1}{2 i \pi} \int_{\left|z-z_{0}\right|=r} F(z) \frac{d z}{z-w}\right\rangle_{B_{0} \cap B_{1}, A_{0}+A_{1}}=\frac{1}{2 i \pi} \int_{\left|z-z_{0}\right|=r}\langle b \mid F(z)\rangle_{B_{0} \cap B_{1}, A_{0}+A_{1}} \frac{d z}{z-w}
$$

The equvalence remains true for $*$-weakly continuous functions.

However, of course, there is no equivalence between (strong) continuity and $*_{\text {-weak }}$ continuity. In the original proof of [3], one made two remaks :

1. Let $1<p_{0} \leq q_{0}<+\infty$ and $1<p_{1} \leq q_{1}<+\infty$, and

$$
\left(\frac{1}{p}, \frac{1}{q}\right)=(1-\theta)\left(\frac{1}{p_{0}}, \frac{1}{q_{0}}\right)+\theta\left(\frac{1}{p_{1}}, \frac{1}{q_{1}}\right) .
$$

If $F$ is an interpolation functor of exponent $\theta$ that satisfies $F\left(L^{p_{0}}, L^{p_{1}}\right)=L^{p}$, then $F\left(\dot{M}^{p_{0}, q_{0}}, \dot{M}^{p_{1}, q_{1}}\right) \subset \dot{M}^{p, q}$. Thus, we have the embeddings of $\left[\dot{M}^{p_{0}, q_{0}}, \dot{M}^{p_{1}, q_{1}}\right]_{\theta, p}$, $\left[\dot{M}^{p_{0}, q_{0}}, \dot{M}^{p_{1}, q_{1}}\right]_{\theta}$ and $\left[\dot{M}^{p_{0}, q_{0}}, \dot{M}^{p_{1}, q_{1}}\right]^{\theta}$ into $\dot{M}^{p, q}$.

2. When moreover $p_{0} / q_{0}=p_{1} / q_{1}=p / q$ we may define for $f \in \dot{M}^{p, q}$ the function $F(z)=\frac{f}{|f|}|f|^{(1-z) \frac{p}{p_{0}}+z \frac{p}{p_{1}}}$. This is a bounded *-weakly continuous function of $z=$ 
$x+i y$ (for $0 \leq x \leq 1$ ) with values in $\dot{M}^{p_{0}, q_{0}}+\dot{M}^{p_{1}, q_{1}}$, holomorphic on the strip

$0<x<1$, with $\sup _{\in \mathbb{R}}\|F(i y)\|_{\dot{M}^{p_{0}, q_{0}}}<+\infty, \sup _{\in \mathbb{R}}\|F(1+i y)\|_{\dot{M}^{p_{1}, q_{1}}}<+\infty$, and $F(\theta)=f$.

If $F$ was strongly continuous, we would find that $f=F(\theta)$ would belong to $\left[\dot{M}^{p_{0}, q_{0}}, \dot{M}^{p_{1}, q_{1}}\right]_{\theta}$. But $F$ is only *-weakly continuous. We may define $G(z)=$ $\int_{0}^{z} F(w) d w$. Then we have $G \in \mathcal{G}$, and $G^{\prime}(\theta)=f$; thus $f$ belongs to $\left[\dot{M}^{p_{0}, q_{0}}, \dot{M}^{p_{1}, q_{1}}\right]^{\theta}$

\section{References}

1. Bergh, J., Löfström, J.: Interpolation Spaces. Springer (1976)

2. Calderón, A.P.: Intermediate spaces and interpolation: the complex method. Stud. Math. 24, 113-190 (1964)

3. Lemarié-Rieusset, P.G.: Multipliers and Morrey spaces. Potential Anal. 38, 741-752 (2013)

4. Ruiz, A., Vega, L.: Corrigenda to "unique continuation for Schrödinger operators" and a remark on interpolation of Morrey spaces. Publ. Math. 39, 404-411 (1995)

5. Sickel, W.: Personnal communication (2014)

6. Zorko, C.T.: Morrey spaces. Proc. Am. Math. Soc. 98, 586-592 (1986) 